CLINICAL STUDY

\title{
Maternal hyperinsulinism and glycaemic status in the first trimester of pregnancy are associated with the development of pregnancy-induced hypertension and gestational diabetes
}

\author{
Simon Kayemba-Kay's ${ }^{1,4}$, Catherine Peters ${ }^{1}$, Michael P P Geary ${ }^{2}$, Nathan R Hill ${ }^{3}$, David R Mathews ${ }^{3}$ \\ and Peter C Hindmarsh ${ }^{1}$ \\ ${ }^{1}$ Developmental Endocrinology Research Group, Clinical and Molecular Genetics Unit, Institute of Child Health, University College London, 30 Guilford \\ Street, London WC1N 1EH, UK, ${ }^{2}$ Department of Obstetrics and Gynaecology, Rotunda Hospital, Dublin 1, Ireland, ${ }^{3}$ Oxford Centre for Diabetes, \\ Endocrinology and Metabolism, Oxford, UK and ${ }^{4}$ Paediatric Endocrinology Unit, Paediatrics and Neonatal Medicine Department, Dreux Hospital, \\ Dreux, France
}

(Correspondence should be addressed to P C Hindmarsh; Email: p.hindmarsh@ucl.ac.uk)

\begin{abstract}
Objective: To evaluate the relationships across a range of glucose and insulin measures at 12 weeks of gestation with the development of pregnancy-induced hypertension (PIH), gestational diabetes mellitus (GDM) and birth size.

Materials and methods: Prospective study of pregnant women booking before 15 th week of gestation. At the first antenatal visit, standard measures of height, weight, blood pressure (BP) and social status were recorded, and blood sample was drawn for measurements of fasting glucose and plasma insulin. Oral glucose tolerance test with $75 \mathrm{~g}$ glucose load was performed after overnight fast. Odds ratios (ORs) with 95\% CI were calculated to determine the risk of developing PIH or GDM depending on quartiles of blood glucose or tertiles of plasma insulin levels.

Results: One thousand six hundred and fifty pregnant women were included in the study. Of them, 1484 delivered a live infant of whom 70 were preterm, 166 did not complete the study, 155 mothers developed PIH (10.4\%), 18 were diagnosed with GDM (1.2\%) and four had both PIH and GDM. At 12 weeks of gestation, women who became hypertensive were heavier $(P<0.001)$, with higher BMI $(P<0.001)$ than controls. Both systolic $(P<0.001)$ and diastolic BPs $(P<0.001)$ were already higher in women who developed PIH. Fasting insulin concentrations were higher in PIH group $(P<0.002)$. Fasting glucose level $>6.8 \mathrm{mmol} / \mathrm{l}$ was associated with the likelihood of delivering a macrosomic baby (OR 3.1 (95\% CI: 1.21-8.0); $P=0.02)$; the effect was heightened in multiparous mothers (OR 4.0 (95\% CI: 1.4-11.1); $P=0.01)$. Fasting plasma insulin had, however, no effect on size at birth in this study. Conclusions: Our data suggest that women who develop PIH may be metabolically challenged at early stages of pregnancy with hyperinsulinism, insulin insensitivity and slightly higher BP.
\end{abstract}

European Journal of Endocrinology 168 413-418

\section{Introduction}

Pregnancy-induced hypertension (PIH) and gestational diabetes mellitus (GDM) are frequent complications affecting $7-10 \%$ and up to $5 \%$ respectively of all pregnancies (1). Both are associated with high maternal and fetal mortality and morbidity risks. PIH is a leading cause of fetal growth restriction and includes preeclampsia. Onset is predominant in the late second or third trimesters of pregnancy. GDM is associated, in the fetus, with an increased risk of adverse perinatal outcomes $(2,3,4)$ and childhood obesity $(5,6)$. Recent data suggest that the risk at mid gestation is represented by a continuous graded relationship with high maternal glucose albeit at concentrations less than those observed in overt GDM (3). In the mother, $20-60 \%$ of those with GDM will go onto develop type 2 diabetes mellitus and an adverse cardiovascular profile $(7,8)$.

Normal pregnancy is a carbohydrate-intolerant state characterised by a progressive increase by the dose response of insulin to glucose $(9,10)$, suggesting that women who are pregnant become insulin resistant with the duration of gestation. Pre-existing hyperinsulinaemia and/or hyperglycaemia has been documented in early or mid pregnancy, before the development of preeclampsia $(11,12)$, gestational hypertension or both $(13,14)$. Women with PIH during the third trimester of pregnancy displayed marked hyperinsulinism in response to an oral glucose tolerance test (OGTT) compared with normotensive controls $(7,8,15)$. 
Whether pregnancy simply serves to amplify tendencies towards higher blood pressure (BP) and GDM remains unclear. If this proposition is the case, then alterations in $\mathrm{BP}$ and glucose parameters within the normal range should be apparent in the early stages of pregnancy in those who may go onto develop $\mathrm{PIH}$ and/or GDM.

We have, therefore, evaluated the relationships across a range of glucose and insulin measures at 12 weeks of gestation with the development of PIH, GDM and birth size in an unselected Caucasian population.

\section{Materials and methods}

Patients were recruited in a prospective manner at first presentation to the Department of Obstetrics and Gynaecology at University College London Hospitals. The study was approved by the Research Ethics Committee of University College London Hospitals, and informed consent for participation was obtained from the mother at the commencement of the study. The inclusion and exclusion criteria for the study have been detailed previously $(16,17)$. Pregnant women were included if they had their first antenatal visit before 20 weeks of gestation, underwent an ultrasound examination demonstrating a structurally normal single fetus and were of Caucasian origin. Women were excluded if they suffered from chronic inflammatory or thrombotic disorders or had a preterm delivery or previous pregnancy complicated by pre-eclampsia, PIH or antepartum haemorrhage or had pre-pregnancy diabetes.

Gestational age was determined from the last date of menstrual period if the date was certain or estimated according to the results of the first obstetric ultrasound. Mothers were recruited in the first trimester (defined as 13 weeks plus 2 weeks to accommodate error on gestational age estimation). Maternal height was measured with a stadiometer (Holtain Limited, Crymych, UK) and recorded to the nearest $0.1 \mathrm{~cm}$. Weight was measured using Seca scales (CMS Weighing Equipment Limited, London, UK) and recorded to the nearest $0.1 \mathrm{~kg}$. BMI was derived from these measures. Current cigarette consumption was categorised as non-smoking, $<10,10-20$ or $>20$ cigarettes/day. Socioeconomic status was determined from age at which full-time education was completed, marital status, occupation and partner's occupation, and social class assignment was made using the classification of the United Kingdom Office of Population Census and Statistics (18).

BP was recorded as the mean of three recordings taken at 1-min intervals after a 5-min rest period using an automated sphygmomanometer (Datascope Accutorr Plus, Birmingham, UK) in a standard room temperature environment of $19-22{ }^{\circ} \mathrm{C}$ with time of day noted. Appropriate-sized adult BP cuffs (extending completely around the arm with a bladder width covering at least two thirds of the upper arm) were used. Hypertension in pregnancy was defined as BP > 140/90 mmHg.

At the first visit to the clinic, a blood sample was drawn from each participant for the measurement of fasting blood glucose and plasma insulin concentrations. An additional routine fasting blood glucose concentration was measured at 28 weeks of gestation. Standard care was provided for all mothers and all were assessed for glucose tolerance during the progression of the pregnancy. The obstetric team caring for the mother was unaware of the results of the early pregnancy blood glucose and plasma insulin results. A provisional diagnosis of gestational diabetes was made if on oral glucose loading dose of $75 \mathrm{~g}$ administered after an overnight ( $8-18 \mathrm{~h}$ ) fast, the 2 -h blood glucose concentrations was $\geq 9.0 \mathrm{mmol} / \mathrm{l}(160 \mathrm{mg} / \mathrm{dl})$, and an actual diagnosis made if there was complete return to normal of the GTT, performed under similar conditions, 6 weeks after delivery.

Birth weight was measured using electronic selfcalibrating scales (Secascales, CMS Weighing Equipment Limited, London, UK) and length by infantometer (Child Growth Foundation, London, UK). Three separate measurements were taken and the mean was recorded. The coefficient of variation (CV) of the measurement error for length was $0.15 \%$ based on ten infants each measured five times.

\section{Glucose and insulin assays}

Blood glucose concentration was measured using a Yellow Springs blood analyser (Yellow Springs, OH, USA). Plasma insulin concentration was measured by RIA (Diagnostic Science Laboratories, Webster, TX, USA), which recognises total insulin. The within-assay $\mathrm{CV}$ values were $8.2,4.8$ and $6.3 \%$ at plasma insulin concentrations of $4.8,17.6$ and $54.6 \mathrm{mU} / \mathrm{l}$ respectively. The between-assay $\mathrm{CV}$ values were 11.2, 9.5 and 6.5 at plasma insulin concentrations of $4.9,16.6$ and $49.7 \mathrm{mU} / \mathrm{l}$ respectively. Standards were calibrated against the WHO RP (83/500). Assay sensitivity was $1.5 \mathrm{mU} / \mathrm{l}$.

\section{Statistical analysis}

All data were explored for the normality of their distribution and $\log$ transformed where appropriate. Birth weight was expressed as a SDS using the UK Growth Reference Data (19) to allow comparison between the sexes and at different gestational ages to account for obstetric preference to deliver at an earlier gestation. Macrosomia was defined as a birth weight $>+2.0$ SDS.

$\chi^{2}$ test was used to compare frequency distributions. Odds ratios (ORs) with 95\% CIs were calculated to 
Table 1 Characteristics at first clinic attendance of the control mothers and those who developed PIH or GDM. Data shown as mean with S.D. in parenthesis.

\begin{tabular}{lcccc}
\hline & $\begin{array}{c}\text { Control } \\
(n=1307)\end{array}$ & $\begin{array}{c}\text { PIH } \\
(n=155)\end{array}$ & $\begin{array}{c}\text { GDM } \\
(n=18)\end{array}$ & P value \\
\hline Age (years) & $31.0(5.6)$ & $30.0(5.7)$ & $32.9(4.6)$ & 0.06 \\
Parity (\%) & 50 & & & \\
0 & 50 & 64.1 & 53.3 & 0.004 \\
$1+$ & $12.9(2.6)$ & $12.6(2.6)$ & $12.5(3.0)$ & 0.27 \\
Gestation at booking (weeks) & $39.5(1.7)$ & $39.0(2.1)$ & $39.3(3.4)$ & 0.008 \\
Gestation at delivery (weeks) & & & & \\
Cigarette smoking (\%) & 80.8 & 84.9 & 80.0 & 0.82 \\
Non-smokers & 19.2 & 15.1 & 20.0 & \\
Smokers & $63.6(11.1)$ & $68.0(14.2)$ & $64.5(11.7)$ & $<0.001$ \\
Weight (kg) & $164.5(6.8)$ & $163.7(6.6)$ & $162.7(7.8)$ & 0.21 \\
Height (cm) & $23.5(4.2)$ & $25.4(5.1)$ & $24.3(3.4)$ & $<0.001$ \\
BMI (kg/m ${ }^{2}$ ) & $105.0(10.5)$ & $110.4(11.8)$ & $106.3(10.4)$ & $<0.001$ \\
Systolic BP (mmHg) & $64.4(7.9)$ & $68.8(8.4)$ & $62.3(7.4)$ & $<0.001$ \\
Diastolic BP (mmHg) & $4.7(0.8)$ & $4.8(1.0)$ & $5.0(1.4)$ & 0.12 \\
Fasting blood glucose (mmol/l) & $13.3(13.5)$ & $16.9(18.3)$ & $13.6(17.0)$ & 0.02 \\
Fasting serum insulin (mU/l) & & & \\
\hline
\end{tabular}

For presentation, the $P$ value refers to ANOVA across the three groups.

determine the risk of developing PIH or GDM depending on blood glucose or plasma insulin concentration. For glucose, the HAPO study cut-points (3) were used for direct comparison whereas for insulin, tertiles were used as not all mothers had insulin measures available. Student's independent $t$-test was used to compare measures between mothers that did not develop PIH and GDM and those that did. BP was adjusted for current size using regression analysis.

\section{Results}

\section{General}

Of the 1790 pregnant women who fulfilled the entry criteria, 1650 (92\%) agreed to participate. Mothers who accepted to participate did not differ from those who refused in terms of pregnancy or pregnancy outcomes. Of the 1650 women, 1484 delivered a live infant of whom 70 were preterm deliveries and 196 developed complications of pregnancy. Of the 166 who did not complete the study, 127 had moved away or been lost to follow-up, 28 had either a miscarriage or termination of pregnancy and 11 withdrew; 155 mothers developed PIH (10.4\%); 18 were diagnosed as GDM (1.2\%); and four mothers had both but because of the small number are not considered further. The remaining 1307 mothers served as a control group. Maternal anthropometric measurements as recorded at the first antenatal attendance were not different from the general UK population. Social class distribution was also similar to that of the UK population with slight preponderance of social class II (class I: 13\%; class II: 41\%; class III: 21\%; class IV: $12 \%$; and class V: $11 \% ; P=0.14)$. Mean maternal age $(P=0.06)$, gestational age at booking $(P=0.27)$ and smoking status $(P=0.82)$ were comparable (Table 1).

\section{Pregnancy-induced hypertension}

At 12 weeks of gestation, women who became hypertensive later in pregnancy were heavier $(P<0.001)$ and had a higher BMI $(P<0.001)$ than controls (Table 1 ). There was a higher percentage of para 0 mothers who went onto develop PIH and a statistically but possibly not clinically significant earlier gestational age at delivery.

Systolic $(P<0.001)$ and diastolic $(P<0.001)$ BPs at the first antenatal visit were already significantly higher in those women who became hypertensive later in pregnancy. This effect remained when BP was adjusted for current body size $(P<0.001)$.

Mean basal fasting blood glucose concentrations were similar between those that did or did not develop PIH whereas fasting plasma insulin concentrations were significantly higher in the PIH group $(P=0.002)$ with a consequent increase in HOMA $\beta$-cell function $(P=0.001)$ and insulin resistance $(P=0.001)$ in $\mathrm{PIH}$ $(646 \pm 48 \%$ and $4.0 \pm 0.4$ respectively) compared with

Table 2 Adjusted odds ratios (ORs) for associations between maternal fasting blood glucose concentration and the development of PIH or GDM.

\begin{tabular}{cccl}
\hline & $\begin{array}{c}\text { Fasting blood } \\
\text { glucose (mmol/l) }\end{array}$ & OR $(95 \% \mathrm{Cl})$ & $\boldsymbol{P}$ value \\
\hline $\mathrm{PIH}$ & $<4.7$ & 1 & \\
& $4.7-5.3$ & $1.22(0.76-1.95)$ & $\mathrm{NS}$ \\
& $5.4-6.0$ & $0.55(0.25-1.22)$ & $\mathrm{NS}$ \\
& $6.1-6.8$ & $2.55(1.16-5.43)$ & 0.02 \\
$\mathrm{GDM}$ & $>6.8$ & $2.28(1.22-4.28)$ & 0.01 \\
& $<4.7$ & 1 & \\
& $4.7-5.3$ & $0.65(0.14-3.03)$ & $\mathrm{NS}$ \\
& $5.4-6.0$ & $1.87(0.50-6.99)$ & $\mathrm{NS}$ \\
& $6.1-6.8$ & $1.77(0.22-14.23)$ & 0.46 \\
& $>6.8$ & $4.62(1.39-15.35)$ & 0.02 \\
\hline
\end{tabular}


Table 3 Adjusted odds ratios (ORs) for associations between maternal fasting plasma insulin concentration and the development of PIH or GDM.

\begin{tabular}{lccl}
\hline & $\begin{array}{c}\text { Fasting plasma } \\
\text { insulin }(\mathrm{mU} / \mathrm{l})\end{array}$ & OR $(95 \% \mathrm{Cl})$ & $\boldsymbol{P}$ value \\
\hline $\mathrm{PIH}$ & $<10.0$ & 1 & \\
& $10-20$ & $0.93(0.56-1.55)$ & $\mathrm{NS}$ \\
$\mathrm{GDM}$ & $>20$ & $1.64(1.05-2.56)$ & 0.02 \\
& $<10.0$ & 1 & \\
& $10-20$ & $0.65(0.14-3.03)$ & $\mathrm{NS}$ \\
& $>20$ & $1.09(0.34-3.50)$ & $\mathrm{NS}$ \\
\hline
\end{tabular}

mothers without PIH $(417 \pm 21 \%$ and $2.9 \pm 0.1$ respectively).

Fasting blood glucose concentrations $>6.1 \mathrm{mmol} / \mathrm{l}$ were associated with an increased risk of developing $\mathrm{PIH}$ (Table 2) as was fasting plasma insulin concentration (Table 3). The insulin effect was heightened when nulliparous mothers were excluded (OR 3.18 (01.357.52); $P=0.009$ ). Combining the glucose and insulin data (Table 4) revealed a higher proportion of mothers with PIH with higher glucose and insulin concentrations.

\section{Gestational diabetes mellitus}

Women who developed GDM were not heavier but had a significantly higher BMI $(P=0.03)$ than those that did not (Table 1). Mean basal fasting blood glucose concentration was higher in those that went onto develop GDM $(P<0.001)$ as was fasting plasma insulin concentration $(P=0.008)$.

Fasting blood glucose concentrations $>6.8 \mathrm{mmol} / \mathrm{l}$ were associated with an increased risk of developing $\mathrm{PIH}$ (Table 2) but fasting plasma insulin concentration was not (Table 3). When analysis was confined to multiparous mothers, plasma insulin concentrations $>20 \mathrm{mU} / \mathrm{l}$ were associated with an increased risk of developing GDM (OR 3.18 (1.35-7.52); $P=0.009$ ). Combining the glucose and insulin data (Table 4) revealed an increased proportion of mothers with GDM as fasting glucose and insulin rose, although this did not achieve statistical significance unless analysis was confined to multiparous mothers where the effect was limited to those with a fasting blood glucose $>6.1 \mathrm{mmol} / \mathrm{l}$ and a plasma insulin either $<20 \mathrm{mU} / \mathrm{l}(7.8 \%)$ or $>20 \mathrm{mU} / \mathrm{l}(4.3 \%)\left(\chi^{2} 8.17\right.$; $P=0.04)$.

A fasting blood glucose concentration $>6.8 \mathrm{mmol} / \mathrm{l}$ was associated with a threefold likelihood of delivering a macrosomic baby (ORs 3.1 (95\% CI: 1.21-8.0); $P=0.02)$ with the effect heightened in multiparous mothers (ORs 4.0 (95\% CI: 1.4-11.1); $P=0.01$ ). Fasting plasma insulin concentration had no statistically significant effect on size at birth in this study. The blood glucose concentration at 28 weeks of gestation did not impact on the likelihood of developing PIH, GDM or macrosomia (Table 5).

\section{Discussion}

These data demonstrate that BP and blood glucose and insulin resistance are already present in those mothers that are destined to develop PIH. The BP differences were within the normal range of pressures. Although it is known that normotensive and essential hypertension women exhibit a fall in their BP in late first to early second trimester of pregnancy, with rise to prepregnancy levels occurring in the third trimester (20), our findings of higher BPs in those women who later developed PIH are consistent with a pre-pathological state. As noted in several studies, women who developed PIH were heavier $(10,11)$ than those that did not and that evidence of hyperinsulinism and insulin resistance was present and associated with the subsequent development of PIH.

Interpreting blood glucose and plasma insulin concentrations in isolation needs to be tempered with the realisation that these two measures change with respect to peripheral insulin sensitivity and $\beta$-cell function and that different concentrations reflect different stages in the evolution of diabetes (21). Hyperinsulinism (fasting plasma insulin concentration $>20 \mathrm{mU} / \mathrm{l}$ ) was associated with a higher prevalence of $\mathrm{PIH}$ and this effect was heightened in those mothers with fasting blood glucose $>6.1 \mathrm{mmol} / \mathrm{l}$ (Table 4 ). These findings are consistent with previous reports linking PIH and insulin resistance $(13,22,23)$. Our findings extend these observations to a much earlier time point in pregnancy before major increases in placental hormones that are known to be insulin antagonistic (24) and suggest that PIH is predisposed, but not exclusively, by higher $\mathrm{BP}$ and hyperinsulinism in the mother antedating the pregnancy.

Although the mechanism that links hyperinsulinism and PIH remains unclear, insulin resistance is sited essentially in peripheral muscle and is probably mediated through the non-oxidative intracellular pathways of glucose disposal $(25,26)$. Insulin per se has direct effects on vascular smooth muscle and epithelium: hyperinsulinaemia increases vascular

Table 4 Interaction between fasting blood glucose and plasma insulin concentrations in the development of PIH or GDM. Data shown as percentages.

\begin{tabular}{lrrrrrr}
\hline & \multicolumn{2}{c}{ PIH } & & \multicolumn{2}{c}{ GDM } \\
\cline { 2 - 3 } \cline { 6 - 7 } Glucose-insulin status & No & Yes & & No & Yes \\
\hline Glucose $<6.1 \mathrm{mmol} / \mathrm{I}$ & 90.6 & 9.4 & & 99.1 & 0.9 \\
$\begin{array}{l}\text { Insulin }<20 \mathrm{mU} / / \\
\text { Glucose }<6.1 \mathrm{mmol} / \mathrm{I}\end{array}$ & 88.9 & 11.1 & & 99.2 & 0.8 \\
$\begin{array}{l}\text { Insulin }>20 \mathrm{mU} / \mathrm{I} \\
\text { Glucose }>6.1 \mathrm{mmol} / \mathrm{I}\end{array}$ & 73.8 & 26.2 & & 97.6 & 2.4 \\
$\begin{array}{l}\text { Insulin }>20 \mathrm{mU} / / \\
\text { Glucose }>6.1 \mathrm{mmol} / /\end{array}$ & 95.7 & 4.3 & & 95.7 & 4.3 \\
$\begin{array}{l}\text { Insulin }<20 \mathrm{mU} / \mathrm{I} \\
\chi^{2}(P \text { value })\end{array}$ & $13.6(P=0.003)$ & & $3.55(P=0.32)$ \\
\hline
\end{tabular}


Table 5 Adjusted odds ratios (ORs) for associations between maternal fasting blood glucose concentration at 28 weeks and the development of PIH or GDM.

\begin{tabular}{cccl}
\hline & $\begin{array}{c}\text { Fasting blood } \\
\text { glucose }(\mathrm{mmol} / \mathrm{l})\end{array}$ & OR $(95 \% \mathrm{Cl})$ & $\boldsymbol{P}$ value \\
\hline $\mathrm{PIH}$ & $<4.7$ & 1 & \\
& $4.7-5.3$ & $1.03(0.71-1.50)$ & 0.54 \\
& $5.4-6.0$ & $0.83(0.71-1.50)$ & $\mathrm{NS}$ \\
& $6.1-6.8$ & $1.55(0.75-2.94)$ & $\mathrm{NS}$ \\
$\mathrm{GDM}$ & $>6.8$ & $2.15(1.0-4.60)$ & 0.05 \\
& $<4.7$ & 1 & \\
& $4.7-5.3$ & $1.6(0.34-8.4)$ & $\mathrm{NS}$ \\
& $5.4-6.0$ & $3.6(0.96-19.6)$ & 0.06 \\
& $6.1-6.8$ & $10.4(2.29-47.4)$ & 0.004 \\
& $>6.8$ & $12.6(2.48-64.1)$ & 0.001 \\
\hline
\end{tabular}

resistance by inducing smooth muscle cell hypertrophy or endothelial dysfunction leading to vasoconstriction and stiffening. Through its action on the sympathic nervous system, it also stimulates the release of epinephrine, thus increasing heart rate and systolic BP $(25,27)$. Indirect effects may operate via the lipid changes that are induced by hyperinsulinaemia that promotes arteriosclerosis and subsequent arterial stiffening and narrowing.

The gestational diabetes findings are consistent with those of the HAPO study (3) with respect to the predictive nature of fasting blood glucose on the development of GDM and outcomes such as macrosomia. Our findings extend those of HAPO to demonstrate that determinants of GDM are present earlier in pregnancy towards the end of the first trimester. There was a fourfold increase in risk of developing GDM if the fasting blood glucose was $>6.8 \mathrm{mmol} / \mathrm{l}$. The impact of fasting insulin was clear but neither blood glucose nor plasma insulin should be considered in isolation. Table 4 highlights this with a steady increase in percentage of mothers with PIH with high plasma insulin concentrations. High blood glucose concentrations appear to amplify the effect. In mothers destined to develop GDM, blood glucose was high and plasma insulin was low, a state suggestive of pancreatic decompensation and consistent with the concept of the failing $\beta$-cell proposed by De Fronzo et al. (21). The effect was more prominent in multiparous mothers, which might imply a reduced ability to increase $\beta$-cell function with subsequent pregnancies. The numbers with GDM in this cohort were lower than might be expected from the literature $(1,6)$, which may reflect the focus in this study on one particular ethnic group and the exclusion of pre-morbid conditions that might impact on fetal growth.

Screening for glucose problems in pregnancy needs to utilise simple tests due to the high throughput of most obstetric units. Our data and those of others $(22,23)$ show that fasting and/or postprandial blood glucose concentrations may not be enough as a proportion of mothers go onto develop PIH from a background of a normal blood glucose concentration but with hyperinsulinism. International recommendations from the 5th International Workshop conference on GDM are focussed essentially on the screening strategy to detect GDM but whether further consideration needs to be given to earlier detection through glucose and insulin measures of a tendency to PIH development needs further consideration.

These data suggest that women who develop PIH in their late pregnancy may be metabolically challenged at the early stages of pregnancy with hyperinsulinism, insulin insensitivity and slightly higher BP in comparison with normotensive controls. The findings and those reported by others are consistent with the view that elements of PIH and GDM have associated glucose and insulin changes already present before pregnancy and that pregnancy may amplify the situation.

\section{Novelty and significance}

The link between insulin resistance and PIH has been described in other studies (ref $(11,12,13,14)$ in the text).

\section{What is new?}

Our study adds to the existing body of evidence that preexisting hyperinsulinism is effectively associated with the development of PIH and GDM. We specifically show that patients likely to develop PIH and GDM could be depicted in early pregnancy by simple tests such as fasting plasma insulin and fasting blood glucose.

\section{What is relevant?}

Our results tend to suggest that preventive measures for a high-risk population can be designed in future with hope to reduce the incidence of PIH and GDM, two conditions that are linked with both maternal and fetal complications.

\section{Declaration of interest}

The authors declare that there is no conflict of interest that could be perceived as prejudicing the impartiality of the research reported.

\section{Funding}

This research did not receive any specific grant from any funding agency in the public, commercial or not-for-profit sector.

\section{References}

1 Cunningham FG \& Lindheimer MD. Hypertension in pregnancy. New England Journal of Medicine $1992 \quad \mathbf{3 2 6} \quad 927-932$. (doi:10.1056/NEJM199204023261405)

2 O'Sullivan JB. Diabetes mellitus after GDM. Diabetes 199140 (Suppl 2) 131-135. 
3 Metzger BE, Lowe LP, Dyer AR, Trimble ER, Chaovarindr U, Coustan DR, Hadden DR, McCance DR, Hod M, McIntyre HD et al. Hyperglycemia and adverse pregnancy outcomes. New England Journal of Medicine 2008358 1991-2002. (doi:10.1056/NEJM oa0707943)

4 Lawrence JM, Contreras R, Chen W \& Sacks DA. Trends in the prevalence of preexisting diabetes and gestational diabetes mellitus among a racially/ethnically diverse population of pregnant women, 1995-2005. Diabetes Care 200831 899-904. (doi:10.2337/dc07-2345)

5 Boerschmann H, Pflüger M, Henneberger L, Ziegler AG \& Hummel S. Prevalence and predictors of overweight and insulin resistance in offspring of mothers with gestational diabetes mellitus. Diabetes Care 201033 1845-1849. (doi:10.2337/ dc10-0139)

6 Lawlor DA, Fraser A, Lindsay RS, Ness A, Dabelea D, Catalano P, Davey Smith G, Sattar N \& Nelson SM. Association of existing diabetes, gestational diabetes and glycosuria in pregnancy with macrosomia and offspring body mass index, waist and fat mass in later childhood: findings from a prospective pregnancy cohort. Diabetologia 201053 89-97. (doi:10.1007/s00125-009-1560-z)

7 Peters RK, Kjos SL, Xing A \& Buchannan TA. Long-term diabetogenic effect of single pregnancy in women with previous gestational diabetes. Lancet 1996347 227-230. (doi:10.1016/ S0140-6736(96)90405-5)

8 McDonald S, Yusuf P, Sheridan SS \& Anand HC. Gerstein for the DREAM Trial Investigators. Dysglycaemia and a history of reproductive risk factors. Diabetes Care 200831 1635-1638. (doi:10.2337/dc08-0621)

9 Bleicher SJ, O'Sullivan JB \& Freinkel N. Carbohydrate metabolism in pregnancy. The interrelations of glucose. Insulin and free fatty acids in late pregnancy and post partum. New England Journal of $\begin{array}{lllll}\text { Medicine } & 1964 & \mathbf{2 7 1} & 866-872 . & \text { (doi:10.1056/ }\end{array}$ NEJM196410222711702)

10 Lind T, Billewicz WZ \& Brown G. A serial study of changes occurring in the oral glucose tolerance test during pregnancy. Journal of Obstetrics and Gynaecology $1973 \quad 801033-1039$. (doi:10.1111/j.1471-0528.1973.tb02976.X)

11 Sermer M, Naylor CD, Gare DJ, Kensole AB, Ritchie JW, Farine D, Cohen HR, McArthur K, Holzapfel S, Biringer A et al. Impact of increasing carbohydrate intolerance on maternal-fetal outcomes in 3637 women without gestational diabetes: the Toronto trihospital gestational project. American Journal of Obstetrics and Gynecology $1995173 \quad 146-156 . \quad$ (doi:10.1016/0002-9378 (95)90183-3)

12 Joffe GM, Esterlitz JR, Levine RJ, Clemens JD, Ewel MG, Sibai BM \& Catalano PM. The relationship between abnormal glucose tolerance and hypertensive disorders of pregnancy in healthy nulliparous women: calcium for preeclampsia prevention (CPEP) study group. American Journal of Obstetrics and Gynecology 1998 179 1032-1037. (doi:10.1016/S0002-9378(98)70210-8)

13 Salomon CG, Graves SW, Greene MF \& Seely EW. Glucose intolerance as predictor of hypertension in pregnancy. Hypertension 199423 717-721. (doi:10.1161/01.HYP.23.6.717)

14 Sowers JR, Sokol RJ, Standley PR, Kruger M, Mason BA, Sowers PS \& Cotton DB. Insulin resistance and increased body mass index in women developing hypertension in pregnancy. Nutrition, Metabolism, and Cardiovascular Diseases 19966 141-146.
15 Bauman WA, Maimen M \& Langer O. An association between hyperinsulininemia and hypertension during the third trimester of pregnancy. American Journal of Obstetrics and Gynecology 1988 $159446-450$.

16 Pringle PJ, Geary MP, Rodeck Ch, Kingdom JC, Kayamba-Kay's S \& Hindmarsh PC. The influence of Cigarette smoking on antenatal growth, birth size and the insulin-like growth factor axis. Journal of Clinical Endocrinology and Metabolism 200590 2556-2562. (doi:10.1210/jc.2004-1674)

17 Kayemba-Kay's S, Geary MP, Pringle J, Rodeck Ch, Kingdom JC \& Hindmarsh PC. Gender smoking during pregnancy and gestational age influence cord leptin concentrations in newborn infants. European Journal of Endocrinology 2008159 217-224. (doi:10.1530/EJE-08-0171)

18 Standard Occupational Classification, vol 3. OPCS. London: Her Majesty's Stationary Office, 1991.

19 Freeman JV, Cole TJ, Chinn S, Jones PRM, White EM \& Preece MA. Cross-sectional stature and weight reference curves for the UK, 1990. Archives of Disease in Childhood $1995 \quad 73$ 17-24. (doi:10.1136/adc.73.1.17)

20 Seely EW \& Salomon CG. Insulin resistance and its potential role in pregnancy-induced hypertension. Journal of Clinical Endocrinology and Metabolism 200388 2393-2398. (doi:10.1210/jc.2003030241)

21 De Fronzo RA, Bonadonna RC \& Ferrannini E. Pathogenesis of NIDDM. A balanced overview. Diabetes Care 199215 318-368. (doi:10.2337/diacare.15.3.318)

22 Schaffir JA, Lockwood CJ. Lapinski R, Yoon L \& Alvarez M. Hypertensive disorders in pregnant women with diabetes. Incidence of PIH among gestational diabetics. American Journal of Perinatology 199512 252-254. (doi:10.1055/s-2007994466)

23 Martinez AE, Gonzales OM, Quninones GA \& Ferrannini E. Hyperinsulinemia in glucose tolerant women with preeclampsia: a controlled study. American Journal of Hypertension 19969 610-614. (doi:10.1016/0895-7061(95)00448-3)

24 Alsat E, Guibourdenche J, Luton D, Frankenne F \& Evain-Brion D. Human placental growth hormone. American Journal of Obstetrics and Gynecology 1997177 1526-1534. (doi:10.1016/S00029378(97)70103-0)

25 Capaldo B, Lembo G, Napoli R, Rendina V, Albano G, Sacca L \& Trimarco B. Skeletal muscle is a primary site of insulin resistance in essential hypertension. Metabolism 199140 1320-1322. (doi:10.1016/0026-0495(91)90036-V)

26 Resnick LM. Cellular ions in hypertension, insulin resistance, obesity and diabetes: a unifying theme. Journal of the American Society of Nephrology 19923 S78-S85.

27 O'Hare JA, Minaker K, Young JB, Rowe JW, Pallotta JA \& Landsberg L. Insulin increases plasma norepinephrine and lowers plasma potassium equally in lean and obese men. Clinical Research $198533441 \mathrm{~A}$.

Received 18 July 2012

Revised version received 11 December 2012

Accepted 14 December 2012 